\title{
Virtual Reality For Microassembly
}

\author{
Martin Probst, Christoph Hürzeler, Ruedi Borer, Bradley J. Nelson \\ Institute of Robotics and Intelligent Systems, ETH Zürich, Switzerland
}

\begin{abstract}
The growing demand for advanced micro-devices that integrate various sensors and actuators, e.g. for biomedical applications, has created a strong need for assembly units that can meet high precision and manipulation requirements. However, developing a sophisticated machine that can fulfill these requirements solves only a part of the problem - having a skilled person that can program and operate the machine must also be addressed. The user interface should provide sufficient information to perform any assembly operation, however, it should also hide or abstract information that would distract the operator from the main task. Controlling the information flow from/to the user and to/from the machine is performed by representing the real environment in a virtual one. This additional layer of abstraction between the user and the machine is based on a standard virtual reality (VR) approach. This paper demonstrates the integration of such a VR system into an existing microassembly station.
\end{abstract}

Keywords: virtual reality, microassembly

\section{INTRODUCTION}

We have previously developed an advanced microassembly system with six degrees of freedom that incorporates a remote center of motion concept[1] (see Figure 1). Three independent cameras with microscopes and an advanced illumination system provide views from different perspectives in order to provide a high resolvability in full 3D while simultaneously allowing the system to avoid occlusions imposed by the gripper. However, even such a multi-viewport design has limitations, e.g. the difficulty to assess distances under certain conditions, as shown in Figure 3. This is particularly problematic if a part has to be precisely aligned or inserted into another. Virtual reality can help in such a situation, because it allows the user to set his viewpoint to any location in space. Since any VR environment is based on a 3D model of the scene, it can also be used for other purposes, such as collision avoidance, visual tracking, etc. In order to synchronize the virtual and the real world, constant visual feedback from the cameras as well as encoder feedback from actuators are processed.

This paper describes the development of such a virtual reality package and its integration into a prototype microassembly system. Section 2 begins with a general overview and analysis of the fields involved. Section 3 considers previous work in this area and Section 4 describes our approach in detail. Experimental results are given in Section 5 and a conclusion and outlook in Section 6.

\section{MOTIVATION}

The advanced six degree-of-freedom microassembly station ${ }^{1}$ previously developed at our institute consists of a two DOF robotic wrist and a four DOF platform that is moved out of the center to a loading position for easier handling of microparts. Together, these axes offer high rotational motion with $\mu m$-precision translation. Various types of end-effectors for different particle dimensions (some with force feedback) and a workbench with an integrated vacuum suction platform provide a versatile environment for handling objects in the microscale (see Figure 1).

Further author information: (Send correspondence to Martin Probst)

Martin Probst: Tannenstrasse 3/CLA H19, 8092 Zürich, E-mail: mprobst@ethz.ch

Christoph Hürzeler: Tannenstrasse 3/CLA H18, 8092 Zürich, E-mail: chuerzel@student.ethz.ch

Ruedi Borer: Tannenstrasse 3/CLA G16.2, 8092 Zürich, E-mail: rborer@ethz.ch

Bradley J. Nelson: Tannenstrasse 3/CLA H15.2, 8092 Zürich, E-mail: bnelson@ethz.ch 

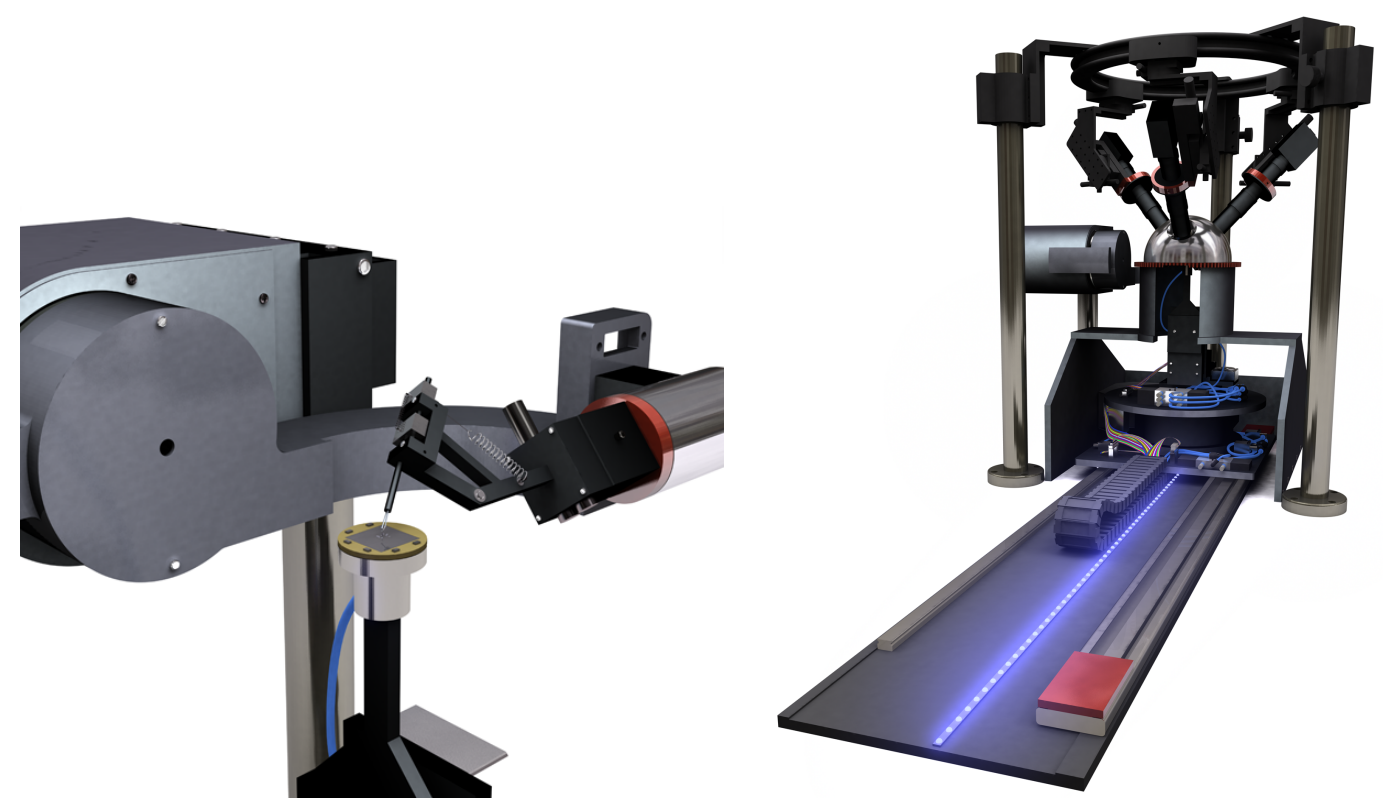

Figure 1. The IRIS Microassembly System V2.

Main Workstation

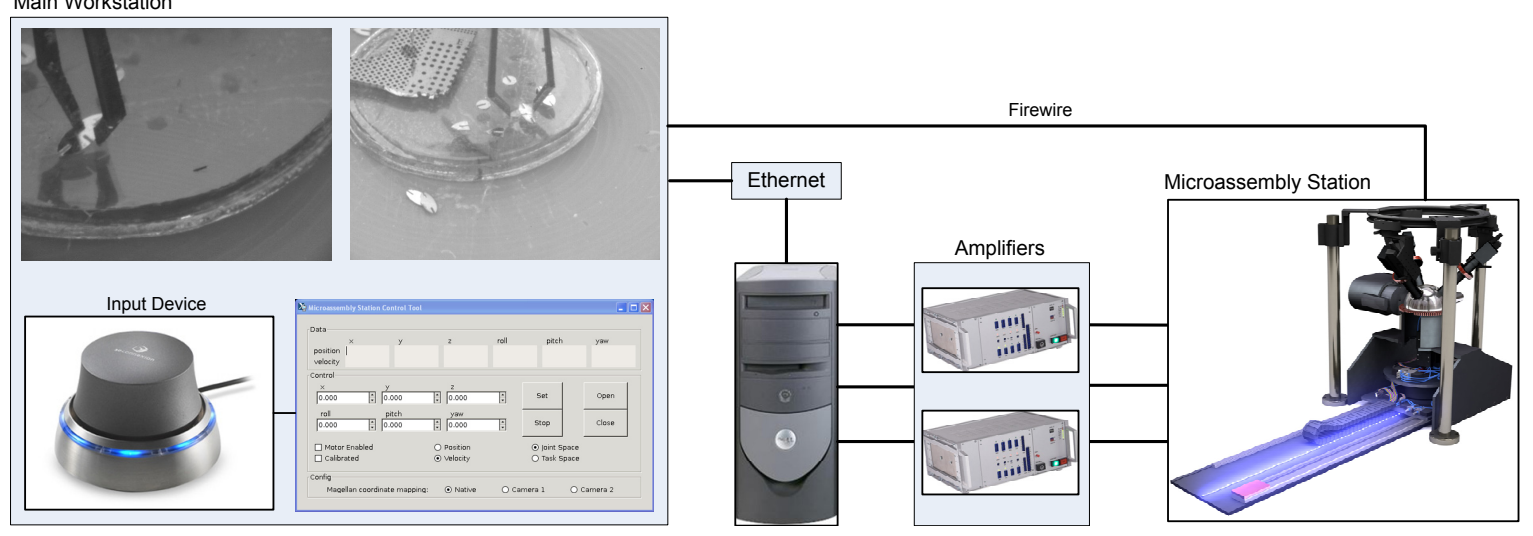

Figure 2. Schematic of a previous microassembly system setup without virtual reality support. Images from two or three firewire cameras surrounding the scene were directly displayed in the control center (see top left) and provided the only information about the manipulation process.

A previous version of the current workcell was attached to a server in which drive amplifiers and position and velocity commands from the host computer were directly transmitted to the server over an ethernet connection (see Figure 2). The host was also directly connected to the cameras, thus providing the only feedback to the user during the assembly process. Several experiments demonstrated the feasibility of the system for assembling microcomponents into complex three-dimensional structures. However, the interpretation of the fixed microscopic views and their translation to world coordinates demand complex spatial orientation skills from the operator. Reflections and bad illumination conditions made the task even more difficult for the operator (see Figure 4).

The ability to change the viewport(s) to any desired location or to have multiple viewports of the same scene for precise alignment tasks is an important advantage over a fixed-viewport system. This ability will also result in significantly reducing operator training time. The additional possibility of hiding certain objects in cluttered scenes and to colorize them according to their function provides additional advantages to the user. 

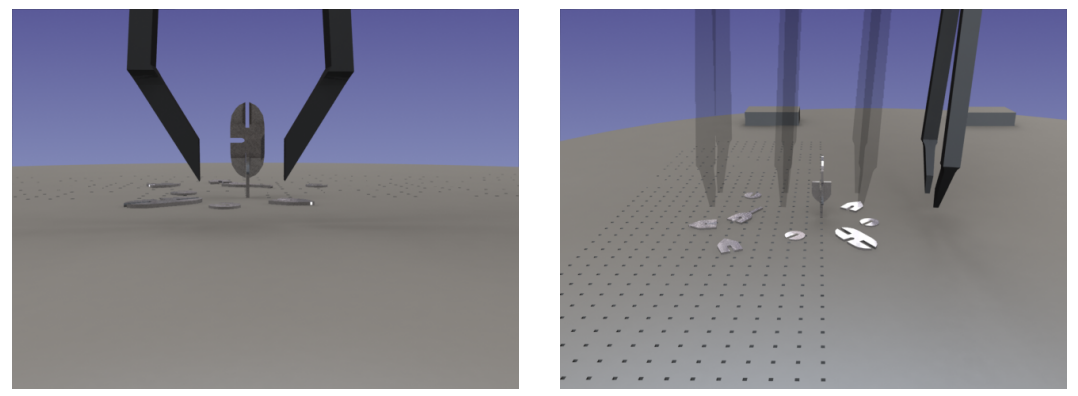

Figure 3. Lack of perspective problem due to the orthographic projection of microscope lenses. The image on the left does not provide any depth information and, thus, hides the effective position of the gripper. Inclining the optical axis with respect to the workbench $\left(45^{\circ}\right.$ in this system) reduces this effect. However, switching the viewport to an oblique position, as shown in the image on the right, provides full depth information.

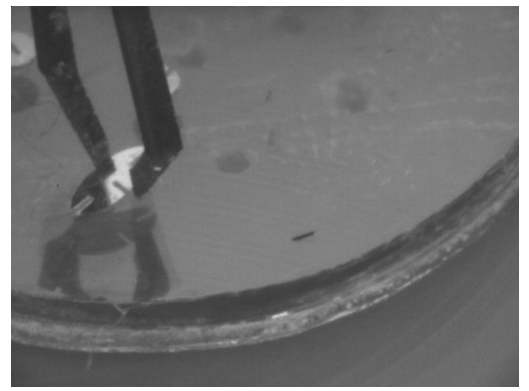

(a)

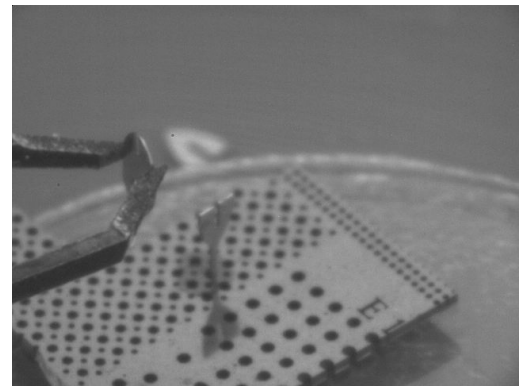

(b)

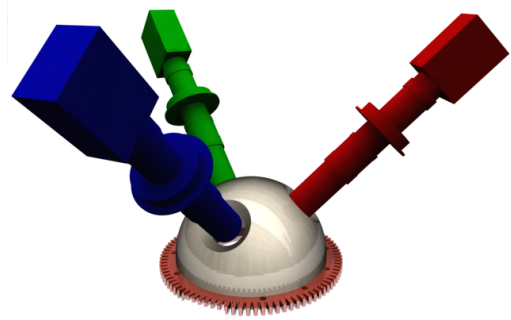

(c)

Figure 4. Assembly of microrobot components given fixed microscopic views. Figures (a) and (b) represent the views from cameras arranged as depicted in Figure (c). The given illumination conditions, shadows, reflections and the cluttered scene create a difficult working environment for the operator.

Furthermore, situations like the perspective view problem as shown in Figure 3 can also be easily overcome by freely customizable viewpoints. Therefore, we believe that abstracting the real world by a virtual one should be a key component for any micromanipulation system. Since any virtual representation is dependent on three dimensional models of all objects in the scene as well as their positions and orientations, a large knowledge base (referred to as common model database throughout this paper) must available. This database can also be used for other purposes. For example, the pose estimation and tracking algorithms used in this context are model-based types, and, thus, require 3D geometry information about the objects to be localized. A common model database can further be used for collision detection and the physical modeling of part interactions. Since most virtual reality packages are based on tree data structures, memberships of objects can be implicitly represented, i.e. part A is currently held by the gripper, lying on the workbench or stuck to another part B.

The primary goal of this work is neither teleoperation (controlling a device over a distance) nor augmented reality (combination of real-world data with virtual elements in order to highlight certain elements). These terms are different from virtual reality (interactive computer simulated environment). However, parts of these concepts might be implicitly used.

\section{LITERATURE REVIEW}

The first reported use of virtual reality for microassmbly was reported by Sulzmann et al. ${ }^{2-5}$ This work was based on a piezo-actuator driven mobile mini-robot equipped with a laser-machined microgripper. The unit was able to grip parts ranging from $20-200 \mu \mathrm{m}$. The robot's workspace was observed by a camera and microscope and vision algorithms extracted position and orientation for updating the virtual reality environment. The approach 
also featured virtual cameras to allow the user to set the viewpoint to arbitrary positions. However, real-time position update was not available. Instead, the virtual world was used for designing assembly tasks which are then simulated. After a successful outcome, the trajectories were transferred to the robot and executed. Since the virtual model was not updated in real-time, safety measures were introduced to the robot controller that prevented the device from collisions.

Two three DOF micro-manipulators with vacuum grippers for objects larger than $10 \mu m$ as well as a camera with a microscope observing the scene were introduced by Alex et al. ${ }^{6}$ The user moved parts by dragging their model in a virtual environment. The scene was then rendered to the same viewpoint as the one of the real camera by transforming 3D features of virtual objects to $2 \mathrm{D}$ screen coordinates and both virtual and real images are compared in image space. The resulting spatial differences were used for the visual servoing control loop.

The microassembly system of ENSIB-University of Orléans ${ }^{7-10}$ consists of two high-precision micro-manipulators each of which is mounted on a coarse motion stage. A micro-conveyer system is used for part feeding and visual feedback provided by two microscopes. A sophisticated simulation package which takes into account predominant forces in the micro-scale is used together with a virtual reality environment for simulating assembly tasks. This powerful software package is shown to be very successful for millimeter-sized objects but reliability suffered due to unpredictable effects at the micro-scale. Human operator supervision is, therefore, still required, and its interaction with an automated control system is investigated in. ${ }^{11}$ In the same context Ammi et al. ${ }^{12}$ realized a manipulation system with an AFM-tip and a microscope. Image processing extracts all features in a scene including dust particles. This information is used as a base for rendering a virtual scene and helps the operator push parts along a collision-free trajectory. A head mounted display as well as a PHANTOM ${ }^{\circledR}$ input device are used for interaction with the operator. Again, physical models are used for the simulation of the interaction forces of all components involved in order to solve for an optimal trajectory.

Tan et al. ${ }^{13}$ present a solution for a peg-in-hole assembly problem using virtual reality support. The setup consists of a three DOF high precision manipulator that is mounted on a six DOF coarse motion stage. Apparently, vision and force feedback are only fed to a PID controller that gets its control input from the operator through a spacemouse, but not to the virtual environment. Instead, virtual reality is used for visualizing the deformation during the insertion process of the pegs.

A more recent publication by Cecil et al. ${ }^{14}$ describes a simple setup with a three DOF base stage, a one DOF manipulator arm and a microscope. Computer vision algorithms implemented in MATLAB are used for tracking gripper and micro-parts in the scene. Additional software components of this experimental setup include an assembly plan manager that reads user defined task descriptions, a collision detection engine, a virtual reality environment as well as a path planner. A motion tracked 3D mouse as well as stereoscopic eyewear are used to fully integrate the operator into the environment.

"Is virtual reality beneficial to microassembly systems? And if it is, what are the advantages and disadvantages. Which tasks can be simplified and which should not be touched? And what are the boundary conditions that have to be taken into account?" Answers to those and other questions are presented in a case study by Zäh et al. ${ }^{15}$ Four test setups and around sixty participants which never had contact with a teleoperation system or virtual environment before were used for a series of experiments. The participants were divided in two groups, one half working with, the other half without, a specific feature to be investigated. The study shows that haptic feedback is a feature that drastically improves control over the assembly process and reduces the probability of damages to microparts. Acoustic feedback can help but not as much as haptic feedback. However, a gain in performance of about one third can be reached with the use of a virtual environment as opposed to a system with classical camera image feedback. Apparently, asynchronous feedback induced by system lag does not impair performance since the brain quickly learns to compensate for delays. This study is particularly interesting, because it is one of the few investigations of the benefits of visual, force and acoustic feedback systems in conjunction with micromanipulation tasks. The results demonstrate that assembly performance is significantly higher, particularly if the information is consistently transmitted using various sensing modalities.

\section{VIRTUAL REALITY ENVIRONMENT}

This section describes the VR environment in detail and highlights its capabilities as well as limitations. The virtual environment is a package that integrates into a larger scale application that is used for controlling the 

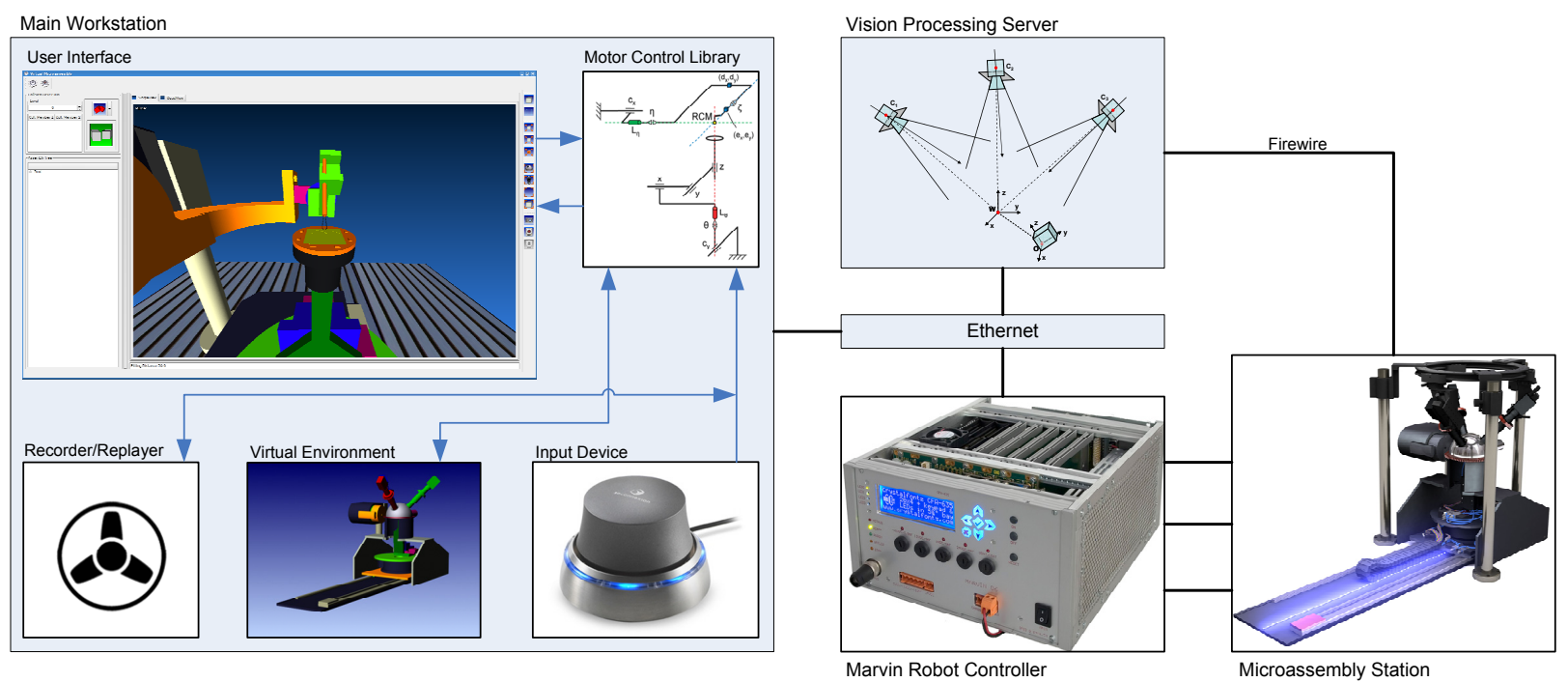

Figure 5. Schematic of the hardware entities of the microassembly system setup. The environment includes three computers: the Marvin robot controller contains all driver electronics and is directly attached to the microassembly hardware. All safety features are also processed here. The vision processing server performs computationally intensive computer vision tasks such as initial pose estimation and tracking. All firewire cameras are directly connected to this machine. Finally, the main workstation communicates with the other two entities over network and provides a user interface with control panels and various input devices, such as space mice, etc.

microassembly system. The primary goal of the software is to control the microassembly station by using a graphical user interface (GUI) and/or an input device. The GUI also contains a virtual reality module that provides an intuitive visual feedback of the real hardware. In addition to this, the system is also capable of recording and replaying manipulation sessions and to locate and track certain components automatically using computer vision.

\subsection{Architecture and Operation Mode}

Figure 5 shows a sketch of the software components for the microssembly system. It consists of three hardware entities: a robot controller (Marvin), a vision server as well as a workstation. The main control applications including input devices as well as the virtual reality environment are performed on the workstation. The dataflow is as follows:

1. Motion events, either from a 3D input device or a task planner, are triggered and sent to the event recorder as well as to the motor control library.

2. The event recorder saves the events to a file so that they can be played back at a later stage. The motor controller library checks the incoming motion commands for consistency and potential collisions by executing them in a virtual reality environment.

3. Upon successful checks, commands are forwarded to the Marvin robot controller over ethernet by a PLAYER $^{16}$ interface and executed on the real hardware.

4. Encoder values from each axis are sent back again through PLAYER to the motor control library that forwards the data to the VR environment for updating the scene for the operator.

This procedure ensures that only valid movements from the operator side are effectively executed, and commands resulting in collisions or area violations can be blocked on the client side. However, this concept of "virtual 


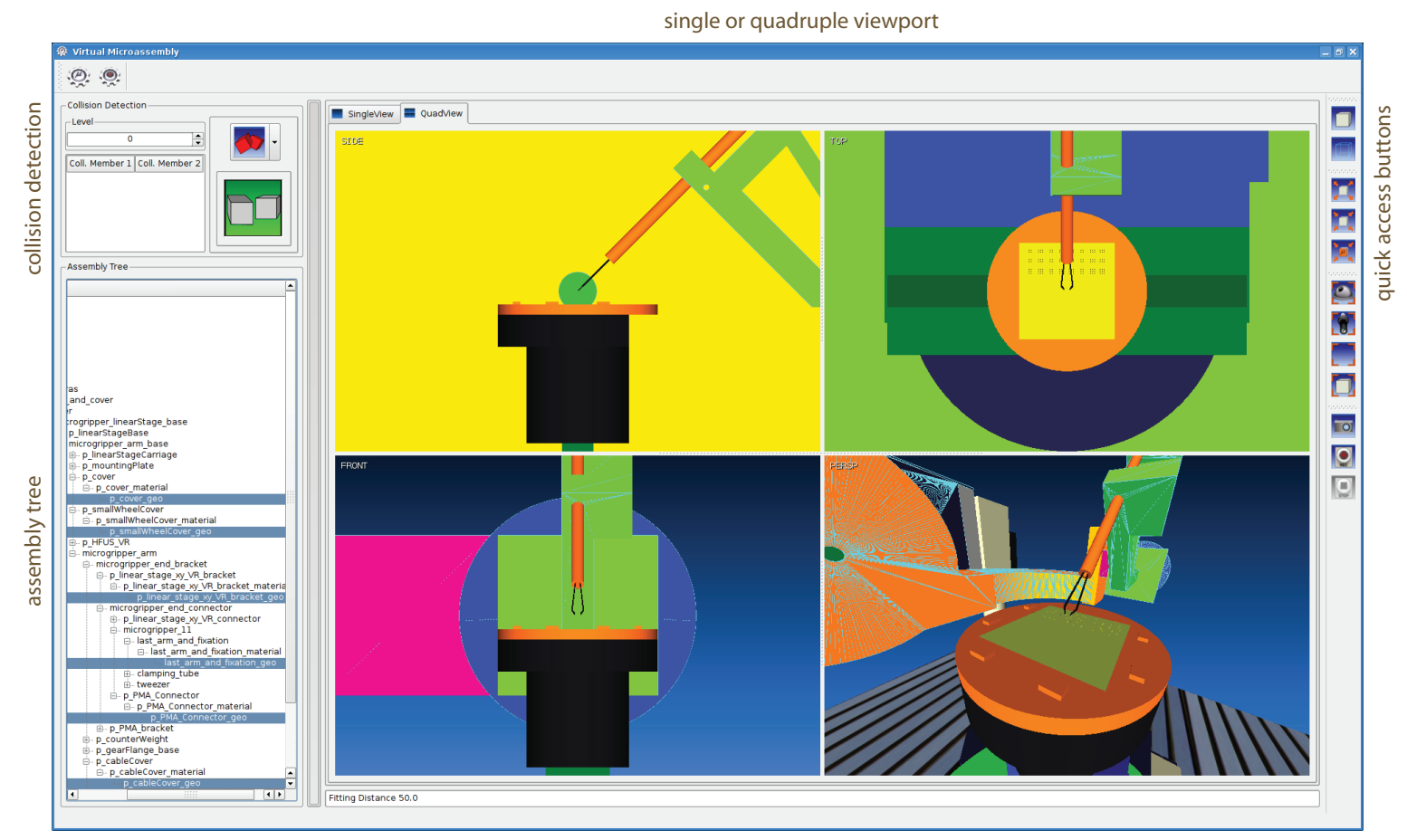

Figure 6. Closeup of the user interface of the virtual reality control center dominated by the large interactive viewport(s) on the right. An assembly tree for hiding individual components as well as a collision detection indicator are arranged on the left.

command execution" also incorporates a lag that potentially makes realtime control unnatural and slows the entire assembly process.

The following sections describe the software components visualization, control center, collision avoidance and event recording running on the workstation.

\subsection{Scene Graph and Visualization}

The visualization component is based on the open source scene graph model OPENSG. A scene graph is a hierarchical representation of 3D geometry, where different nodes at each leave describe geometry, transformation, lights, etc. Camera nodes are used to define arbitrary viewports which are rendered by an OPENGL rendering pipeline in this case. In order to setup a scene graph, 3D model data of the environment is required. A computer aided design (CAD) model is readily available from the development process but has to be converted to a supported format by the scene graph package. This multi-step process is complicated by the large number of $3 \mathrm{D}$ file formats on the market. Since commercial conversion programs are often prohibitively expensive, additional digital content creation (DCC) software is used to process CAD model data so that the data can be readily imported. The model complexity is first reduced by removing screws, holes, blends, etc. in the CAD software itself. This modified dataset is then exported to the STL file format and imported in MAYA for post-processing. The repositioning of pivot points to the tool center point as well as the assignment of materials to functional groups is then performed. Finally, the virtual model is exported to a VRML2 file that retains hierarchical information and can be directly imported by the open scene graph loader module.

The capabilities needed for the user interface required the development of a set of modules that directly operate on the scene graph. This set includes functionalities such as node location and selection, node labeling, node grouping, manipulation of materials (e.g. solid or wireframe display), node hiding, graph navigation, etc. Up to this point the common model database contains a hierarchical tree and a large set of tools. Next, this information is displayed in a graphical user interface (GUI) as shown in Figure 6. The window center is 


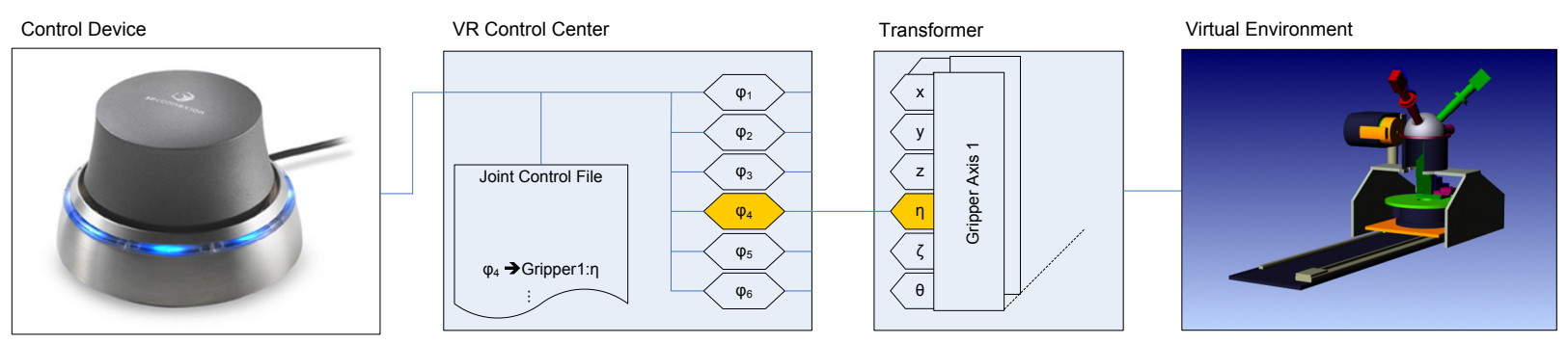

Figure 7. Signal flow from the input device to the virtual reality scene. Motion commands from an input device are sent to the VR control center and mapped to axes of transformation nodes based on the configuration in the joint control file.

dominated by four customizable viewports or a single large one. Quick access buttons for zooming, hiding the most important component groups, as well as for taking screenshots and movies are arranged in the bar on the right. The left area shows the component hierarchy that is useful for navigation and hiding/unhiding of parts. The main window also contains a collision detection control widget.

\subsection{VR Control Center}

The core functionality and flexibility of the virtual reality environment is provided by the VR control center module. It basically handles and dispatches the user input according to definitions that can be changed offline. Figure 7 shows the signal flow. A configuration file (joint control file) specifies which input device axes corresponds to which transformation node axes. It is also possible to define limits of motion for each joint. Integration over time and filtering of the input velocity commands is also required since the transformation nodes require position information. This architecture has the important advantage that any virtual device can be easily controlled since it only requires the adaption of the scene graph itself as well as the joint control file.

\subsection{Collision Avoidance}

Collision avoidance is a crucial component in this environment, as already pointed out in Section 4.1. Position, velocity or trajectory commands are first executed in the virtual reality environment, checked for collisions and are only passed to the hardware if checks are passed. The collision detection library CoLLDET used in this system has been developed by Zachmann et al. ${ }^{17,18}$ from Clausthal university and directly operates on an OpenSG scene graph. This library has a collision pipeline that itself contains one or more collision callbacks that are sequentially called upon execution. Each collision callback is built from a collision pair that denotes two geometrical nodes in the scene graph that should be checked as well as a callback function that is invoked upon collision and returns details about the colliding entities. If colliding objects are detected, motion command(s) to the robot controller are not sent thus preventing damage to the hardware.

Collision detection is a computationally intensive task, and, therefore, adds a lag to the system. However, the concept of collision pairs as opposed to including the entire scene tree offers the possibility of dramatically reducing consumption. In this case, six collision pairs are used for the gripper and workbench. Further pairs are added for microparts. The lag time is also a function of the complexity of the objects. Thus, computation time can be further reduced by good preprocessing of the CAD model before importing it to the scene graph.

\subsection{Event Recording/Replaying and Task Planning}

This environment also includes an event recording and replaying module (see Figure 5). This can be helpful for repetitive tasks, a task planning system or demonstrations. If desired, the input events are provided with a timestamp and dumped to a Python file. This can be loaded and executed at anytime for an exact reproduction of motion. So far, only input device (i.e. 3D spacemouse) events have been considered, but the final goal is to integrate any possible command so that the full functionality of the machine is available for recording and replaying. 
This concept can also be used for a task planning system. The definition of higher level commands, such as "moveTo(x,y,z,roll,pitch,yaw)", would allow writing assembly plans and executing them virtually before running them on the real hardware.

\subsection{Synchronization}

This virtual environment has to precisely represent the position, orientation and status of the real microassembly hardware, as it is the only feedback provided to the operator. Thus, bringing those two entities to congruence and keeping them that way is important. Our setup uses a two step process. When the machine is started, the Marvin motor controller independently performs a calibration of all axes and drives them to known positions. The transformation nodes of the scene graph are then adapted accordingly to match the real hardware. Assuming that all drives perform as specified and return correct encoder values, there is no imminent need for re-syncing the two worlds.

\section{PERFORMANCE}

As already briefly explained in Section 1, the microassembly system V2 has a full six DOF manipulator, three microscopic lenses with cameras, an advanced illumination dome as well as a part feeding mechanism. The manipulator itself is divided in two halves: a four DOF base system with the working platform that provides $x, y, z$ and yaw motion and a two DOF arm with the remaining roll and pitch axes. DC and stepper motors as well as harmonic drives are used for high precision movements in the sub-micron range.

Figure 8 shows the results of the performance of the six DOF manipulator. A velocity command $c_{v}$ is generated by a $3 \mathrm{D}$ space mouse, processed by the virtual reality environment as explained in the previous sections and sent to the motor controller over ethernet (see Figure 5 for clarification of the dataflow). The resulting position measurement $m$ is sent back to the workstation for updating the scene graph. For comparison, the integral of the velocity command $c_{v}$ is also displayed. In order to compensate for the low pass filter characteristics of the whole information chain, the input signal has been low pass filtered with a cutoff frequency of $f_{c}=50 \mathrm{~Hz}$. The reaction of the system to the input commands feels natural and the results are also within expectance. The lag between command and result is around $0.5 \mathrm{~s}$ which is acceptable for a reasonable workflow. One reason for the lag is certainly the broadcast-type network connection. Another is the calculation of the next position combined with a collision check. Computers with higher performance as well as optimizing the network should further reduce this effect. The figures also show that the hardware omits certain commands. The reason for that lies in the structure of the PLAYER robot controller which, in its current setting, only keeps the most current command. This mode ensures a more direct hardware control at the cost of missing commands.

\section{CONCLUSION}

We have presented the architecture and components of a sophisticated virtual reality software environment for the use in a six DOF microassembly station. The main goal was the simplification and improvement of the assembly procedure in order to create a device that can be operated with little training effort. The system also solves the lack of perspective problem and provides a database that can be used for collision detection as well as model-based computer vision algorithms. It is based on the OpenSG scene graph model and consists of various modules for different tasks, such as visualization, collision detection, input device abstraction, etc. Its open design allows it to be used for other tasks or mechanical setups. Performance tests indicate promising results and demonstrate the feasibility of the concept. One of the biggest challenges is synchronization, i.e. making sure that the virtual reality is a precise copy of the real machine. This becomes even more crucial at the microscale, where insertion tasks require high precision movements. Calibrating the manipulator and evaluating its encoder feedback is straightforward and fairly failsafe. However, the visualization of the microparts in the virtual environment requires accurate position and orientation information under all circumstances. Localization and tracking modules will be integrated next in order to provide this information. Further optimization on the communication level is planned in order to reduce the lag between motion commands and machine reaction. 


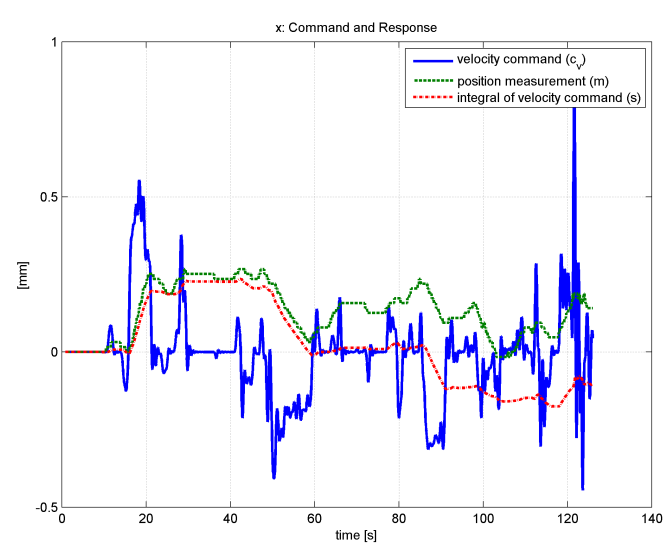

(a)

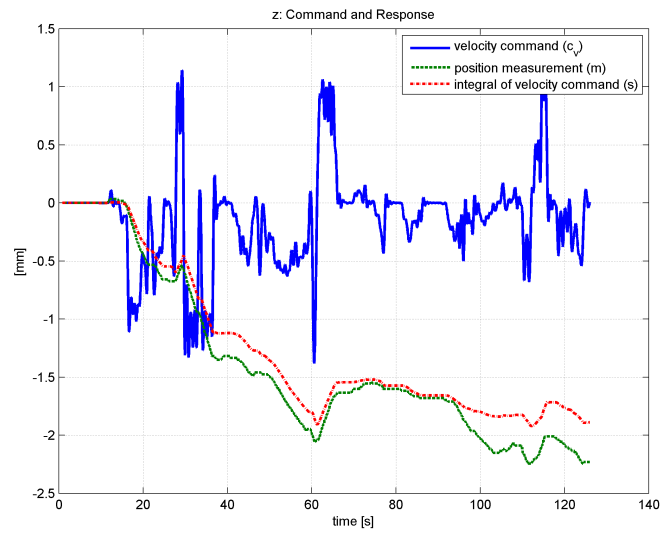

(c)

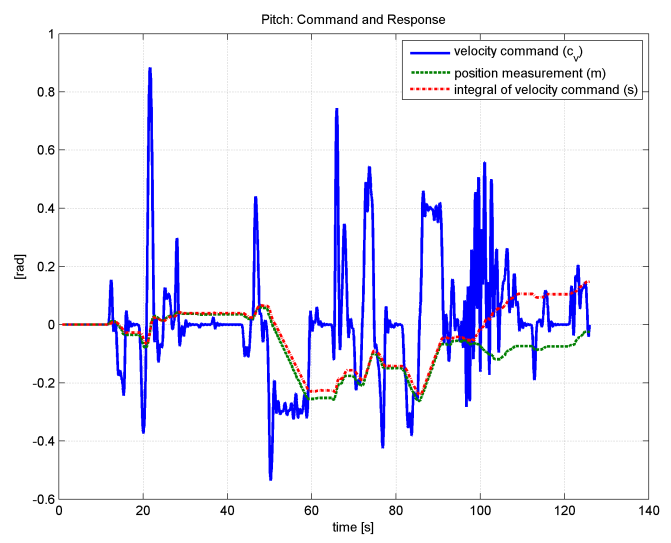

(e)

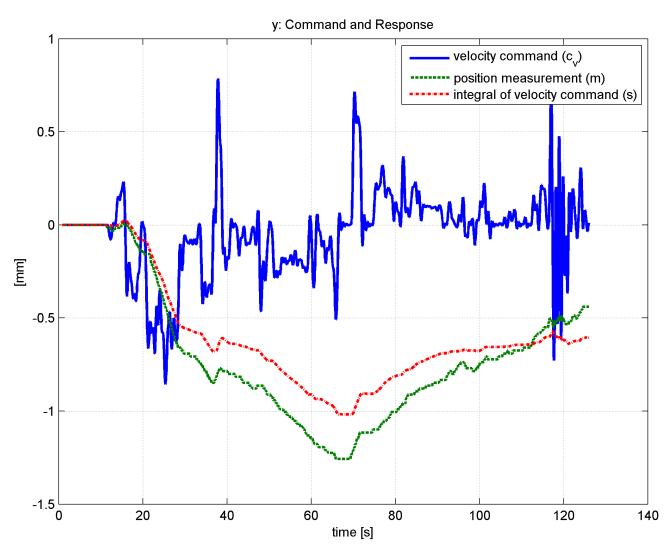

(b)

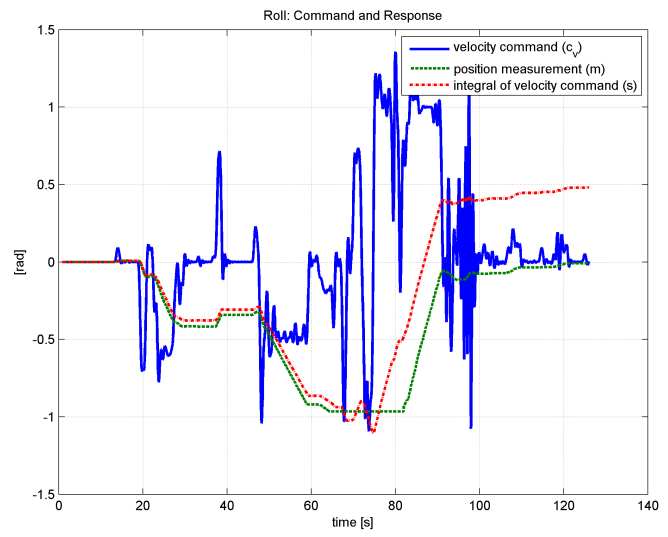

(d)

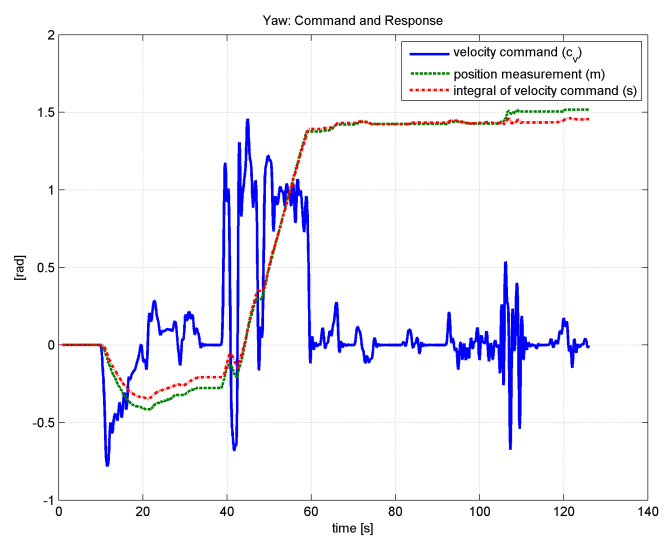

(f)

Figure 8. Velocity commands $c_{v}$ from an input device (3D space mouse) sent to the motor controller, its integral $s(t)=\int_{t=0}^{x} c_{v} d t$ and the measured position feedback of the main axes $x, y, z$, roll, pitch, yaw. The input velocity commands have been filtered using a Butterworth lowpass filter $\left(n=9, f_{c}=50 \mathrm{~Hz}\right)$. 


\section{REFERENCES}

1. M. Probst, K. Vollmers, B. E. Kratochvil, and B. J. Nelson, "Design of an advanced microassembly system for the automated assembly of bio-microrobots," in Proc. 5th International Workshop on Microfactories, Oct. 2006.

2. A. Sulzmann and J. Jacot, "3d computer graphics based interface to real microscopic worlds for \&robot telemanipulation and position control," in IEEE International Conference on Systems, Man and Cybernetics. 'Intelligent Systems for the 21st Century'., 1, pp. 286-291vol.1, Oct. 22-25 1995.

3. A. Sulzmann, J.-M. Breguet, and J. Jacot, "Microvision system (MVS): a 3D computer graphic-based microrobot telemanipulation and position feedback by vision," Proc. SPIE Photonics East 2593, pp. 38-49, 1995.

4. A. Sulzmann, J. Carlier, and J. Jacot, "Distributed Microscopy: towards a 3D computer graphic-based multi user microscopic manipulation, imaging and measurement system," Proc. SPIE Photonics East 2902, 1996.

5. A. Sulzmann, J. Carlier, and J. Jacot, "Virtual Reality and high-accuracy vision feedback as key information for microrobot telemanipulation," Proc. SPIE Photonics East Proc 2906, 1996.

6. J. Alex, B. Vikramaditya, and B. Nelson, "Teleoperated micromanipulation within a VRML environment using Java," in Proc. IEEE/RSJ International Conference on Intelligent Robots and Systems, 3, pp. 1747$1752,1998$.

7. C. Cassier, A. Ferreira, and S. Hirai, "Combination of vision servoing techniques and VR-based simulation for semi-autonomous microassembly workstation," in Proc. IEEE International Conference on Robotics and Automation ICRA 'O2, 2, pp. 1501-1506, 2002.

8. A. Ferreira, C. Cassier, and S. Hirai, "Automatic microassembly system assisted by vision servoing and virtual reality," IEEE/ASME Transactions on Mechatronics 9(2), pp. 321-333, 2004.

9. M. Hamdi and A. Ferreira, "Microassembly planning using physical-based models in virtual environment," in Proc. IEEE/RSJ International Conference on Intelligent Robots and Systems (IROS 2004), 4, pp. 3369$3374,2004$.

10. M. Ammi, A. Ferreira, and J.-G. Fontaine, "Virtualized reality interface for tele-micromanipulation," in Proc. IEEE International Conference on Robotics and Automation ICRA '04, 3, pp. 2776-2781, 2004.

11. A. Ferreira, "Strategies of human-robot interaction for automatic microassembly" in Proc. IEEE International Conference on Robotics and Automation ICRA '03, 3, pp. 3076-3081, 2003.

12. M. Ammi and A. Ferreira, "Path planning of an AFM-based nanomanipulator using virtual force reflection," in Proc. IEEE/RSJ International Conference on Intelligent Robots and Systems (IROS 2004), 1, pp. 577$582,2004$.

13. F. Tan, L. Sun, W. Rong, and J. Zhu, "A collision response method in virtual environment of peg-inhole microassembly," in Fifth World Congress on Intelligent Control and Automation (WCICA 2004), 4, pp. 3210-3213 Vol.4, 2004.

14. J. Cecil, D. Powell, and D. Vasquez, "Assembly and manipulation of micro devices-a state of the art survey," Robotics and Computer-Integrated Manufacturing 23, pp. 580-588, Oct. 2007.

15. M. Zäh, B. Petzold, O. Anton, M. Ehrenstraer, and J. Schilp, "Telepräsenz und Teleaktion in der Mikrosystemtechnik: Systeme zur Analyse und Bewertung zu unterstützender Fertigkeiten," in Proc of HCRS, pp. 9196, 2002.

16. B. Gerkey, R. Vaughan, and A. Howard, "The player/stage project: Tools for multi-robot and distributed sensor systems," in Proceedings of the International Conference on Advanced Robotics (ICRA 2003), pp. 317323, June 30 - July 32003.

17. G. Zachmann, "Minimal hierarchical collision detection," in Proc. ACM Symposium on Virtual Reality Software and Technology (VRST), pp. 121-128, (Hong Kong, China), Nov. 11-13 2002.

18. G. Zachmann and R. Weller, "Collision detection library using OpenSG." Source Code, 2007. http://cg.in.tuclausthal.de. 